FERMILAB-TM-2693-AD

\title{
Fermilab Accelerator Complex: Status and Improvement Plans
}

\author{
Mary Convery, Michael Lindgren, Sergei Nagaitsev and Vladimir Shiltsev
}

Fermi National Accelerator Laboratory, PO Box 500, Batavia, IL 60510, USA

\begin{abstract}
Fermilab carries out an extensive program of accelerator-based high-energy particle physics research, which relies on $8 \mathrm{GeV}$ and $120 \mathrm{GeV}$ proton accelerators. Routine operations with 700 $\mathrm{kW}, 120 \mathrm{GeV}$ proton beam power on a neutrino target was achieved in 2017. There are plans to further increase the beam power to the $900 \mathrm{~kW}$ range. The next major upgrade of the Fermilab accelerator complex, the PIP-II project, is under development. Its goal is to deliver 1.2 MW of beam power on target at the start of the DUNE experiment in the middle of the next decade. PIP-II will replace the existing 40-year old $400 \mathrm{MeV}$ normal-conducting Linac with a modern $800 \mathrm{MeV}$ superconducting RF based linear accelerator. There are several concepts for future upgrades that will increase the beam power to $>2.4 \mathrm{MW}$, after replacement of the existing 8 $\mathrm{GeV}$ Booster synchrotron. The Muon Campus provides $3.1 \mathrm{GeV}$ polarized muons to the $\mathrm{g}-2$ experiment and will provide $8 \mathrm{~kW}$ of $8 \mathrm{GeV}$ protons to the Mu2e production target. The Fermilab Test Beam Facility provides a primary $120 \mathrm{GeV}$ proton beam of moderate intensity, (1-300 kHz) and secondary particle beams of energies down to about $1 \mathrm{GeV}$, consisting of hadrons, muons and/or electrons/photons for detectors tests. In this article we discuss the current performance of the Fermilab accelerator complex, the upgrade plans for the next two decades, and the accelerator R\&D program to address cost and performance risks for these upgrades.
\end{abstract}




\section{Introduction: Overview of the Fermilab accelerator complex}

The Fermilab accelerator complex (see Fig. 1) is one of the largest in the world, and consists of $16 \mathrm{~km}$ of accelerators and beamlines, three high power targets, several low power target stations, and many experimental and service buildings. It delivers beams of protons and secondary particles. The beam originates from a pulsed duoplasmatron $\mathrm{H}$ - ion source followed by a $750 \mathrm{keV}$ RFQ and a $400 \mathrm{MeV}$ normal-conducting pulsed linac that injects into an $8 \mathrm{GeV}$ rapid-cycling-synchrotron (RCS) Booster, which is largely an original construction ca 1960's. The Booster combined function dipole magnets operate in a $15 \mathrm{~Hz}$ resonant circuit, which sets a fundamental clock for the complex. Historically, not all cycles could be loaded with protons due to limitations from injection, extraction, and RF system and beam loss. The next machine downstream in the complex is the Recycler, a $3.3 \mathrm{~km} 8 \mathrm{GeV}$ storage ring made from permanent magnets. Originally built for storage and accumulation of low intensity antiproton beams during the Tevatron Collider Run II (2001-2011) [1], the Recycler is now used to stack high intensity protons for loading into the $120 \mathrm{GeV}$ Main Injector synchrotron, which is located in the same tunnel. The circumference of the Recycler is sufficient to accommodate six batches of 84 Booster bunches each; however, through the technique called "slip-stacking", six more batches can be injected in the machine in addition to six slightly decelerated original batches, making the total of 12 batches. These batches travel at slightly different velocities and "slip" with respect to each other until they overlap and at that moment they are transferred to the Main Injector and accelerated to the maximum energy (more details on the "slip-stacking" method can be found in [1]). The Fermilab proton accelerator complex supports a number of experiments - e.g., the $400 \mathrm{MeV}$ Linac beam is sent to the Mucool Test Area, $8 \mathrm{GeV}$ protons from the Booster are supplied to the $8 \mathrm{GeV}$ Booster Neutrino Beam (BNB), ANNIE, MicroBooNE, MiniBooNE, MITPC, ICARUS (near future), and SBND (future), and to the "muon g-2" and "Mu2e" (near future) muon experiments. The $120 \mathrm{GeV}$ proton beam from the Main Injector supports neutrino experiments at NuMI (MINERvA, NOvA) and DUNE in the future, as well as the fixed target experiments SeaQuest and test beam facility. See Ref.[2] for detailed information on these experiments. The Fermilab accelerator complex has delivered neutrino beam with $>85 \%$ uptime on average over the past 5 years.

In May of 2014, the Particle Physics Project Prioritization Panel (P5), advisory to the Office of High Energy Physics in the US Department of Energy, released a report [3] that identified the top priority for the U.S.-hosted high-energy physics program to be a high energy neutrino program to determine the mass ordering and measure $\mathrm{CP}$ violation, based on the Fermilab accelerator complex, upgraded for increased proton intensity. The current long baseline neutrino program utilizes the Neutrinos from the Main Injector (NuMI) beam line that was built to provide protons for the MINOS experiment, located in the Soudan Mine in Minnesota, $725 \mathrm{~km}$ away. Later, the NOvA experiment was built $810 \mathrm{~km}$ away in Ash River, Minnesota. It also uses the NuMI beam line, but it is built $14.6 \mathrm{mrad}$ off axis, producing a narrower neutrino energy spread, resulting in an improved resolution for the $\mathrm{CP}$ violating phase and mass hierarchy. The physics goal of the future long baseline neutrino program set forth by the P5 report is: “...a mean sensitivity to CP violation 
of better than $3 \sigma \ldots$ over more than $75 \%$ of the range of possible values of the unknown CPviolating phase $\delta_{\mathrm{CP}}$ ". To this end, a new beam line and experiment are being planned. The beam line is the Long Baseline Neutrino Facility (LBNF) [4] at FNAL and the new experiment is the Deep Underground Neutrino Experiment (DUNE) [5], located in the Sanford Underground Research Facility (SURF) near Lead, South Dakota, $1300 \mathrm{~km}$ away from Fermilab. The P5 physics goals require about $900 \mathrm{kt} \cdot \mathrm{MW} \cdot$ years of exposure (product of the neutrino detector mass, average proton beam power on the neutrino target and data taking period). Assuming a 40 kton Liquid Argon detector, this would take over 50 years at the $400 \mathrm{~kW}$ beam intensity which was typical when the program was first conceived. For this reason, a series of accelerator upgrades toward the eventual goal of multi MW proton beam power have been undertaken and planned.

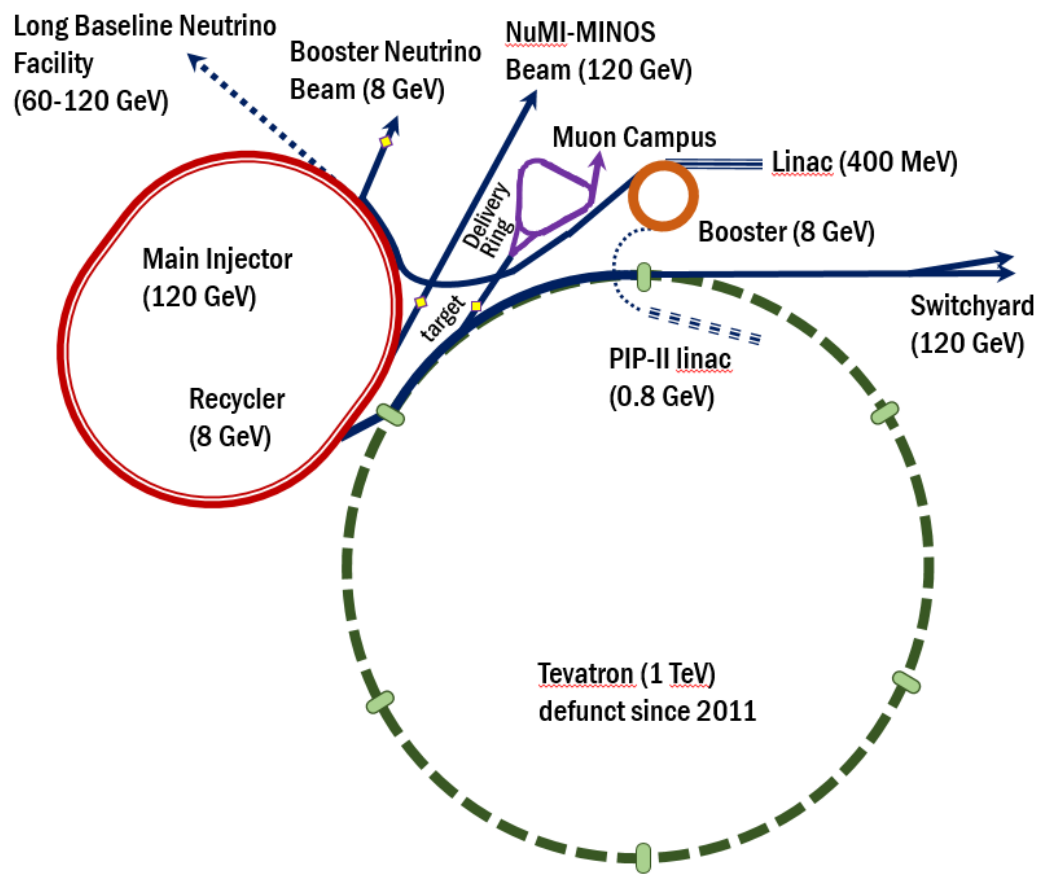

FIG.1: Fermilab accelerator complex includes $400 \mathrm{MeV} \mathrm{H}$ - pulsed normal-conducting RF linac, $8 \mathrm{GeV}$ proton Booster synchrotron, $8 \mathrm{GeV}$ Recycler storage ring that shares a tunnel with the $120 \mathrm{GeV}$ proton Main Injector synchrotron, and a $3.1 \mathrm{GeV}$ muon Delivery Ring. A number of beamlines connect the accelerators, bring the beams to fixed targets and to various high energy physics experiments. The most notable future additions (dotted lines) include the LBNF beam line for DUNE and $0.8 \mathrm{GeV}$ CW-capable SRF PIP-II linac located inside the Tevatron ring and the corresponding beamline for injection into the Booster.

\section{Current upgrade activities}

In 2014-2018 the Fermilab accelerators underwent several upgrades to maximize the proton output from the existing complex, with an ultimate goal of delivery of $700 \mathrm{~kW}$ of $120 \mathrm{GeV}$ beam power from the Main Injector. The key elements were the reduction of losses and upgrades of the pulsed RF hardware in the Booster to allow beam to be accelerated on all $15 \mathrm{~Hz}$ cycles. This goal has recently been achieved and the total proton output from the Booster achieved $2.1 \cdot 10^{17}$ 
protons per hour. In addition, commissioning of the $6+6$ batch slip-stacking in the Recycler reduced the Main Injector cycle time the from $2.2 \mathrm{~s}$ of the MINOS/Collider Run II era to $1.33 \mathrm{~s}$. Due to these improvements, in early 2017 the Main Injector achieved a world-record of $716 \mathrm{~kW}$ average proton beam power over one hour to the NuMI beam line - see Fig.2. On the way, the operations team increased the number of batches slip-stacked in the Recycler in steps (just 6 batches in late 2014, then $2+6,4+6$ and, finally, $6+6$ batches in mid-2016). At each step, the increase in intensity was followed by tuning for efficiency and minimization of beam losses. Installation of the Recycler beam collimation system and commissioning of a more efficient beam feedback system to control coherent instabilities during the slip-stacking process were the keys to the intensity increase.

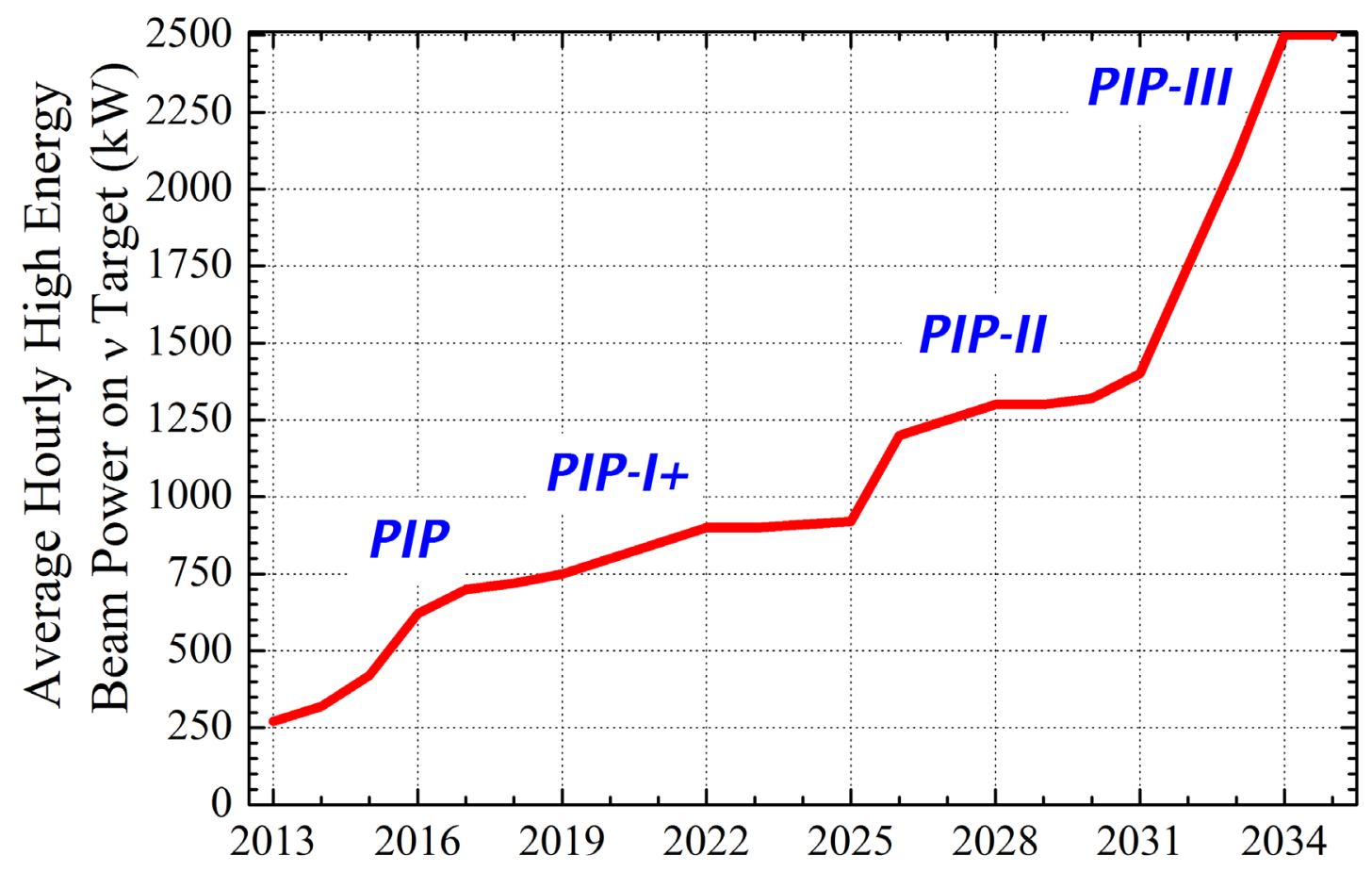

FIG.2: Average hourly average $120 \mathrm{GeV}$ proton beam power from the Main Injector synchrotron on neutrino target - past accomplishments (2013-2018) and future plans (PIP-II) and concepts (PIP-III).

The Fermilab accelerator operations team is currently considering a campaign aimed at increasing the NuMI beam power to the $900 \mathrm{~kW}$ range, without major changes to the existing accelerator complex. The campaign needs to be finished prior to the construction of the PIP-II linac (see next chapter) and includes

1. Upgrades of the NuMI target station to be robust up to $1 \mathrm{MW}$.

2. Loss reduction in the Booster, and a $30 \%$ increase in protons per pulse - see Fig.3a.

3. Increasing the Booster rate from $15 \mathrm{~Hz}$ to $20 \mathrm{~Hz}$. 
4. Modification of the Recycler slip-stacking RF system and the Main Injector ramp to allow faster $1.1 \mathrm{~s}$ cycle.

\section{Proton Improvement Plan - II (PIP-II)}

The key feature of the proposed Proton Improvement Plan-II (PIP-II) project [6] is to replace the existing $400 \mathrm{MeV}$ normal-conducting RF pulsed linear accelerator with a new $800 \mathrm{MeV}$ machine based on superconducting RF cavities, capable of CW operation. It will allow an increase of the $120 \mathrm{GeV}$ proton beam power available to the new LBNF beamline to $1.2 \mathrm{MW}$. In addition, the Booster rate will be increased from 15 to $20 \mathrm{~Hz}$, allowing full Main Injector beam power to be achieved at the lower energy of $60 \mathrm{GeV}$ (vs current $120 \mathrm{GeV}$ ), and $80 \mathrm{~kW}$ of beam for the $8 \mathrm{GeV}$ neutrino program - see full list of the PIP-II key technical parameters in the Table 1. PIP-II linac will be part of eventual extension of beam power to LBNF/DUNE to more than 2 MW (PIP-III) and will also provide a flexible platform for long-range development of the Fermilab complex; in particular, provide an upgrade path for a factor of 10 increase in beam power to the Mu2e experiment, and for extension of accelerator capabilities to include flexible high-bandwidth pulse formatting/high beam power operations.

Table 1. Beam parameters of the present Booster and PIP-II upgrades.

\begin{tabular}{|l|l|l|l|}
\hline Performance Parameter & Present & PIP-II & \\
\hline Linac Beam Energy & 400 & 800 & $\mathrm{MeV}$ \\
\hline Linac Beam Current & 25 & 2 & $\mathrm{~mA}$ \\
\hline Linac Beam Pulse Length & 0.03 & 0.6 & $\mathrm{msec}$ \\
\hline Linac Pulse Repetition Rate & 15 & 20 & $\mathrm{~Hz}$ \\
\hline Linac Beam Power to Booster & 4 & 18 & $\mathrm{~kW}$ \\
\hline Booster Protons per Pulse & $4.3 \times 10^{12}$ & $6.5 \times 10^{12}$ & \\
\hline Booster Pulse Repetition Rate & 15 & 20 & $\mathrm{~Hz}$ \\
\hline Booster Beam Power @ 8 GeV & 80 & 160 & $\mathrm{~kW}$ \\
\hline Beam Power to 8 GeV Program (max; MI @ 120 GeV) & 32 & 80 & $\mathrm{~kW}$ \\
\hline Main Injector Protons per Pulse & $4.9 \times 10^{13}$ & $7.6 \times 10^{13}$ & \\
\hline Main Injector Cycle Time @ 60-120 GeV & 1.33 & $0.7-1.2$ & $\mathrm{sec}$ \\
\hline LBNF/NuMI Beam Power @ 60-120 GeV & 0.7 & $1.0-1.2$ & $\mathrm{MW}$ \\
\hline LBNF Upgrade Potential @ 60-120 GeV & NA & $>2$ & $\mathrm{MW}$ \\
\hline
\end{tabular}

The PIP-II project received CD-1 approval from the U.S. Department of Energy in 2018 and is scheduled for completion in 2025-2026. The project has an extensive R\&D program focused on reduction of the technical risk and the total project cost via development of the superconducting (SC) RF cavities, research towards significant improvement of the quality factor of the SC RF 
cavities and construction and test of the PIP-II low-energy front-end. The PIP-II SC RF cavity development has five different types, operating at three different frequencies:

1. 162.5 MHz Half-Wave Resonators (HWRs).

2. $325 \mathrm{MHz}$ Single-Spoke Resonators (SSR1 and SSR2).

3. $650 \mathrm{MHz}$ Low and High-Beta resonators.

The high- $Q_{0} \mathrm{SC}$ RF studies discovered that nitrogen doping during the $\mathrm{Nb}$ cavity surface processing [7] more than doubles the cavity's quality factor $Q_{0}$ and reduces the required cryogenic capacity. Fast cooling of the cavities (which operate at $2 \mathrm{~K}$ ) enhances the magnetic flux expulsion out of the SC cavity [8] and improves $Q_{0}$. The front-end Linac test facility [9] will demonstrate the two most challenging PIP-II design elements: very low energy transition from "room-temperature" RF acceleration to "cold" SC RF at $2.1 \mathrm{MeV}$, and a $162.5 \mathrm{MHz} \mathrm{CW}$ beam chopper. The PIP-II Injector Test facility has recently achieved beam acceleration through its $2.1 \mathrm{MeV} C W \mathrm{RFQ}$.

\section{The Muon Campus}

The Muon Campus was constructed using infrastructure from the former Tevatron antiproton source. It is currently designed to operate in two modes, one for the Muon g-2 experiment, and the other for the future Mu2e experiment. In both modes, a bunch of $4 \times 10^{12} 8 \mathrm{GeV}$ protons from the Booster is re-bunched using 2.5 MHz RF in the Recycler into four bunches of $10^{12}$ protons with $95 \%$ of the beam contained in a 120 ns time window.

For the g-2 experiment, sixteen of these bunches are extracted to the g- 2 target every $1.4 \mathrm{~s}$ for an average of $11.4 \mathrm{~Hz}$, in bursts of 8 bunches at $100 \mathrm{~Hz}$. The target and lithium lens used for focusing secondary particles are reused from the antiproton source. A pulsed dipole magnet selects a 3.1-GeV secondary beam, which is transported roughly $200 \mathrm{~m}$ to the Delivery Ring. On the fourth turn around the $500 \mathrm{~m}$ circumference Delivery Ring, the muons captured from forward pion decays are separated in time from the secondary protons, allowing a kicker with fast risetime to remove the protons. For every $10^{12}$ protons on target, $O\left(10^{5}\right)$ muons with $>95 \%$ polarization and momentum $3.094 \mathrm{GeV} / \mathrm{c} \pm 2 \%$ are delivered to the $\mathrm{g}-2$ muon storage ring. A similar number of positrons accompany the muons but do not interfere with the experiment. Full operation of g-2 experiment began in 2018.

In the Mu2e mode, $8 \mathrm{GeV}$ protons will bypass the $\mathrm{g}$ - 2 target station and will be transported to the Delivery Ring, from which they are resonantly extracted over a spill length of $43 \mathrm{~ms}$ to the production target inside the Mu2e solenoid. An extinction system using an AC dipole will remove out-of-time protons on target at the level of $10^{-10}$ or better. Commissioning of beam to $\mathrm{Mu} 2 \mathrm{e}$ experiment is expected to begin in 2020, with beam to the target in 2023.

\section{Fermilab Test Beam Facility}


The Main Injector accelerates the beam to $120 \mathrm{GeV}$ at a frequency of $53 \mathrm{MHz}$, at which point a process called Resonance Extraction is started and a fraction of the beam is resonantly extracted to the Fermilab Test Beam Facility in a slow spill for each Main Injector rotation. The facility has two areas - MTest and MCentral. The primary beam consists of high-energy protons $(120 \mathrm{GeV})$ at moderate intensities $(1-300 \mathrm{kHz})$. This beam can also be targeted to create secondary particle beams in MTest areas of energies down to about $1 \mathrm{GeV}$, consisting of hadrons, muons and/or electrons/photons. Experiments in these areas typically turn over on a weekly (1-4 weeks) basis. The MCentral areas are purposed more towards long-term experiments with a turnover rate of months or even years. This beamline has the same secondary particle beams as MTest, but has the added capability of a tertiary beam line. The tertiary beam line can produce hadrons down to energies of $200 \mathrm{MeV}$.

\section{FAST facility}

The Fermilab Accelerator Science and Technology (FAST) facility's mission is to enable a broad range of beam-based experiments to study fundamental limitations to charged particle beam intensity and to develop transformative approaches to particle-beam generation, acceleration and manipulation [10]. It is fully-equipped to support R\&D for the next generation of particle accelerators and incorporates a $40 \mathrm{~m}$ circumference Integrable Optics Test Accelerator (IOTA) storage ring with the capability of storing either electrons or protons with up to $150 \mathrm{MeV} / \mathrm{c}$ momentum. It has two injector accelerators: a 150-300 MeV photoinjector-based superconducting RF linear accelerator of electrons and $2.5 \mathrm{MeV}$ RFQ accelerator of protons $(\mathrm{H}+)$ from a duoplasmatron source. The electron injector employs one $1300 \mathrm{MHz}$ ILC-type cryomodule powered by a $10 \mathrm{MW}$ klystron, modulator, and an RF power distribution system that allows tests with up to 5 $\mathrm{Hz}$ repetition-rate. Both electron and proton injectors can be augmented with additional beam lines, dumps, and test areas to support user research and tests beyond those on the integrable optics and space-charge compensation envisioned in the IOTA ring.

The facility provides $20 \mathrm{MeV}$ electron beams since 2015 and $50 \mathrm{MeV}$ electrons since 2016. In 2017, the entire $300 \mathrm{MeV}$ electron injector was fully commissioned to meet the IOTA beam specifications and demonstrated a world-record beam accelerating gradient of $31.5 \mathrm{MV} / \mathrm{m}$ in 1.3 $\mathrm{GHz}$ superconducting cavities. Starting in 2018, $100 \mathrm{MeV}$ electron beams are being injected into and circulated in the IOTA ring. Proton injector beam commissioning is scheduled for 2019-2020. In 2018, the IOTA multi-year accelerator science research program began. It is focused on beam tests of novel ideas such as non-linear integrable optics, space-charge compensation with electron lenses, optical stochastic cooling and studies of beam instabilities and Landau damping that are needed for the next generation of multi-MW proton beams, including a potential PIP-III project [11 - 14]. Fermilab will also strive to establish a national user facility for accelerator science based on the FAST facility and its IOTA ring that will contribute to advances in all fields that use accelerators: light sources; free-electron lasers; very-high-energy colliders; electron-ion colliders, and high-intensity machines. 


\section{Summary}

Fermilab's program is focused on supporting accelerator-based neutrino research as the top priority for the U.S.-hosted high-energy physics program. The accelerator-based neutrino research program relies on the operation of the $120 \mathrm{GeV}$ proton beamlines for NuMI at present, and for the DUNE experiment when it starts, in the middle of the next decade. Routine operation with a world-record $700 \mathrm{~kW}$ of average high-energy beam power on the neutrino target has been achieved in 2017, and a further increase to the $900 \mathrm{~kW}$ range is anticipated after a series of continuing improvements to the existing accelerator complex in the coming years. The next major upgrade of the FNAL accelerator complex, the PIP-II project, aims at 1.2 MW beam power on the neutrino target at the start of the DUNE experiment. It will replace the existing $400 \mathrm{MeV}$ pulsed normalconducting Linac with a modern $800 \mathrm{MeV}$ superconducting RF linear accelerator. The accelerator research program is exploring several concepts to replace the existing $8 \mathrm{GeV}$ Booster and further double the beam power to $>2.4 \mathrm{MW}$ after the PIP-II project. An extensive accelerator R\&D program with significant international contributions has been launched to address cost and performance risks for these upgrades: the PIP-II Injector Test facility, development of costeffective SC RF cavities, and an experimental R\&D program at the IOTA ring to demonstrate novel space-charge mitigation methods. Fermilab welcomes international contributions to the LBNF beamline design and construction, the PIP-II SRF linac and auxiliary systems, R\&D on multimegawatt targets and cost-effective SRF technology as well as actively develops collaboration on the fundamental high-brightness proton beams physics program at IOTA.

\section{References}

[1] V.Lebedev and V.Shiltsev (eds.) Accelerator Physics at the Tevatron Collider (Springer, 2014).

[2] http://news.fnal.gov/fermilab-at-work/experiments-and-projects/

[3] Building for Discovery Strategic Plan for U.S. Particle Physics in the Global Context, P5 report (June 2014); available at http://www.usparticlephysics.org/

[4] http://lbnf.fnal.gov/

[5] DUNE Collaboration, Long-Baseline Neutrino Facility (LBNF) and Deep Underground Neutrino Experiment (DUNE) Conceptual Design Report, arXiv:1601.05471

[6] The PIP-II Reference Design Report (2015) https://indico.fnal.gov/getFile.py/access?resId=0\&materialId=2\&confId=9939

[7] A.Grassellino, et. al., Supercond. Sci. Technol., 26, 102001 (2013, Rapid Communication).

[8] A.Romanenko, et. al., Appl. Phys. Lett. 105, 234103 (2014).

[9] A.Shemyakin, Proc. of IPAC2013, 1093 (2013).

[10] S.Antipov, et al, JINST 12, T03002 (2017).

[11] E.Prebys, et. al., Proc. of IPAC2016, 1010 (2016).

[12] V.Shiltsev, Mod. Phys. Lett. A 32 (16), 1730012 (2017).

[13] S.Nagaitsev, V.Lebedev, FERMILAB-PUB-18-546 (2018).

[14] J.Eldred, A.Valishev, Proc. of IPAC2018, 894 (2018). 